\title{
Geometrical control of ionic current rectification in a configurable nanofluidic diode
}

\author{
Mohammad Amin Alibakhshi, ${ }^{1}$ Binqi Liu, ${ }^{2}$ Zhiping $\mathrm{Xu},{ }^{3}$ \\ and Chuanhua Duan ${ }^{1, a)}$ \\ ${ }^{1}$ Department of Mechanical Engineering, Boston University, Boston, Massachusetts 02215 , \\ USA \\ ${ }^{2}$ School of Aerospace Engineering, Tsinghua University, Beijing 100084, China \\ ${ }^{3}$ Applied Mechanics Laboratory, Department of Engineering Mechanics and Center \\ for Nano and Micro Mechanics, Tsinghua University, Beijing 100084, China
}

(Received 14 May 2016; accepted 24 August 2016; published online 7 September 2016)

\begin{abstract}
Control of ionic current in a nanofluidic system and development of the elements analogous to electrical circuits have been the subject of theoretical and experimental investigations over the past decade. Here, we theoretically and experimentally explore a new technique for rectification of ionic current using asymmetric 2D nanochannels. These nanochannels have a rectangular cross section and a stepped structure consisting of a shallow and a deep side. Control of height and length of each side enables us to obtain optimum rectification at each ionic strength. A 1D model based on the Poisson-Nernst-Planck equation is derived and validated against the full 2D numerical solution, and a nondimensional concentration is presented as a function of nanochannel dimensions, surface charge, and the electrolyte concentration that summarizes the rectification behavior of such geometries. The rectification factor reaches a maximum at certain electrolyte concentration predicted by this nondimensional number and decays away from it. This method of fabrication and control of a nanofluidic diode does not require modification of the surface charge and facilitates the integration with lab-on-a-chip fluidic circuits. Experimental results obtained from the stepped nanochannels are in good agreement with the 1D theoretical model. Published by AIP Publishing. [http://dx.doi.org/10.1063/1.4962272]
\end{abstract}

\section{INTRODUCTION}

Nanofluidic channels with dimensions comparable to the Debye screening length portray peculiar transport characteristics due to the effect of surface charge. Ion transport in such systems has been under extensive studies due to their relevance in understanding the activity of biological ion channels, ${ }^{1-3}$ as well as the prospect of exploiting them in biomedical and chemical applications. Over the past decade, there has been a wealth of studies focusing on the design of nanofluidic components analogous to solid-state elements, and in particular, efforts have been made to design nanofluidic transistors, ${ }^{4-9}$ diodes, ${ }^{10-38}$ and an inductor ${ }^{39}$ as the key elements for ionic circuits. These elements have been widely used for sensing, separation, and concentration of biomolecules such as nucleic acids, proteins and DNA ${ }^{8-12}$ single cell analysis, ${ }^{4}$ and precise control of the transport of ions and charged molecules. ${ }^{5-7,10,13-41}$

Rectification of ionic current in nanofluidic channels occurs as a result of an asymmetry in the system. Unlike a symmetric nanofluidic channel which acts analogous to a solid-state resistor, an asymmetric nanochannel gives rise to a fluidic diode. A nanochannel with asymmetric geometry, ${ }^{14-23}$ asymmetric surface charge distribution along the nanochannel, ${ }^{24-31}$ asymmetric electrolyte concentration ${ }^{3,32}$ or $\mathrm{pH}^{1,3}$ at the nanochannel terminals, or asymmetric concentration

${ }^{\text {a) }}$ Author to whom correspondence should be addressed. Electronic mail: duan@bu.edu 
polarization ${ }^{33,34}$ conducts ionic current preferentially in one direction and inhibits the ionic current in the opposite direction. Each of these rectification methods have been employed and extensively studied by researchers in the past decade. Yet, utilization of these transport phenomena in nanofluidic channels is hampered by difficulties in their controlled design and fabrication as well as their integration in larger fluidic circuits. The existing rectification methods based on conical nanopipettes/nanopores, ${ }^{13-21}$ bipolar membrane systems, ${ }^{35-37}$ or asymmetric membranes ${ }^{22,23}$ have limited control over their geometries and are very difficult to integrate in 2D lab-on-a-chip devices. On the other hand, nanofluidic channels with patterned surface charge as the symmetry breaker have been shown to effectively rectify the ionic current. ${ }^{24-31}$ Nevertheless, this method requires access to and surface modification of only certain part of a nanochannel, which may not be easily possible in larger fluidic circuits. In addition, some of the surface modification methods are subject to decay over time which may limit the diode's life time to as short as several hours. An alternative method is applying a gate voltage along certain part of the nanochannel which makes the fabrication more complicated. ${ }^{31}$ In this paper, we present a technique for rectifying current that is solely based on geometry control and does not require any surface modification, and yet can be easily designed, fabricated, and integrated in a larger circuit with more components. The theoretical background necessary for understanding the rectification behavior is presented as a 1D numerical model, and the numerical results are compared and validated against experimental results and a good agreement is observed. This nanofluidic diode can be configured to yield optimum current rectification at electrolyte concentration of interest.

\section{THEORETICAL BACKGROUND}

\section{A. Problem description}

The problem geometry is schematically shown in Fig. 1. The nanochannel has a stepped structure and is divided into two parts with length $L_{1}$ having a height of $h_{1}$ and length $L_{2}$ at height $h_{2}$. Width of both parts of the nanochannel is $w$, and without loss of generality let us assume $h_{2}>h_{1}$. The Poisson-Nernst-Planck equation correlating charge density and electrostatic potential can be employed to solve this problem

$$
\begin{gathered}
\nabla^{2} \varphi=\frac{-\sum z_{i} n_{i} e}{\varepsilon \varepsilon_{0}}, \\
\frac{\partial n_{i}}{\partial t}+\mathbf{u} . \nabla n_{i}=\nabla \cdot\left(D_{i} \nabla n_{i}+n_{i} \frac{D_{i} z_{i} e}{k_{b} T} \nabla \varphi\right),
\end{gathered}
$$

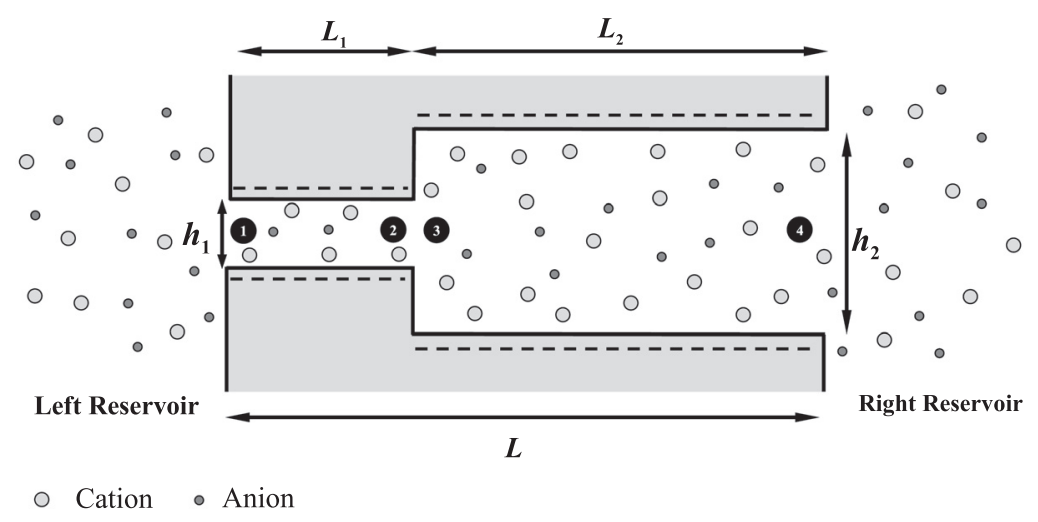

FIG. 1. The nanochannel geometry consisting of a shallow and a deep side with rectifying property. 
where $n$ is the concentration of species with the index $i$ referring to either of cations or anions, $D_{i}$ is the diffusivity of species, $k_{b}$ is the Boltzmann constant, $T$ is the temperature, $z_{i}$ is the valence, $e$ is the elementary charge, $\varphi$ is the electric potential, and $\mathbf{u}$ is the velocity vector. Assuming the electroosmotic flow is negligible $(\mathbf{u}=0)$ and for steady state condition the Nernst-Planck equation can be further simplified. One should note that it is not trivial that electroosmotic flow can be neglected for all nanochannel heights and electrolyte concentrations. ${ }^{6,42}$ Nevertheless, for the sake of simplicity here these terms are neglected and a complementary discussion will be presented in Section IV. These equations can be numerically solved using a Finite element Method (FEM) to yield the current as a function of the applied voltage for the geometry of interest. Alternatively, a 1D model can be constructed based on these equations ${ }^{26}$ which is easy to solve without a finite element package, and facilitates parametric study of the system under investigation. This system of equation for monovalent ions $\left(\left|z_{i}\right|=1\right)$ with similar diffusion coefficient $D$ for both cations and anions is written as (see the supplementary material)

$$
\begin{aligned}
& \bar{N}_{2}^{2}-1=\bar{N}_{3}^{2}-\frac{1}{\tau^{2}}, \\
& \bar{\varphi}_{2}-\bar{\varphi}_{3}=\ln \frac{\bar{N}_{2}+1}{\bar{N}_{3}+\frac{1}{\tau}}, \\
& \bar{M} l=-\left(\bar{N}_{2}-\bar{N}_{1}\right)+\left(\bar{\varphi}_{2}-\bar{\varphi}_{1}\right), \\
& \bar{M}(1-l)=-\left(\bar{N}_{4}-\bar{N}_{3}\right)+\left(\bar{\varphi}_{4}-\bar{\varphi}_{3}\right), \\
& l=\left(\frac{\bar{N}_{1}}{\bar{M}}+\frac{\bar{I}}{\bar{M}}\right)\left[1-\exp \left(\frac{\bar{\varphi}_{2}-\bar{\varphi}_{1}}{\bar{I}}\right)\right]+\frac{1}{\bar{M}}\left(\bar{\varphi}_{2}-\bar{\varphi}_{1}\right), \\
& 1-l=\left(\frac{\bar{N}_{3}}{\bar{M}}+\frac{\bar{I}}{\bar{M}}\right)\left[1-\exp \left(\frac{\bar{\varphi}_{4}-\bar{\varphi}_{3}}{\bar{I}}\right)\right]+\frac{1}{\bar{M}}\left(\bar{\varphi}_{4}-\bar{\varphi}_{3}\right) .
\end{aligned}
$$

In this equation, the nondimensional values of $\bar{x} \bar{\varphi}, \bar{N}, \bar{M}, \bar{I}, \tau$ are defined as

$$
\bar{x}=\frac{x}{L}, \quad \bar{\varphi}=\frac{\varphi}{k_{b} T / e}, \quad \bar{N}=\frac{N}{2 \sigma / e h_{1}}, \quad \bar{M}=\frac{M}{2 \sigma D / e L}, \quad \bar{I}=\frac{I}{M}, \quad \tau=\frac{h_{2}}{h_{1}},
$$

with $N=n_{+}+n_{-}, l$ being the length ratio $\left(l=\frac{L_{1}}{L}\right)$, and the indices " $1-4$ " referring to different locations along the nanochannel as shown in Fig. 1. Moreover, $M$ and $I$ are two constants proportional to the mass flow rate and the current $(i=I w e)$ that based on shallow side quantities can be written as $M=-h_{1} D \frac{d N}{d x}+\frac{2 \sigma D}{k_{b} T} \frac{d \varphi}{d x}$ and $I=-\frac{N h_{1} D e}{k_{b} T} \frac{d \varphi}{d x}$. It is important to note that the concentration and the electric potential at the entrance of the nanochannel (points 1 and 4 in Fig. 1) are different from the reservoirs values. However, assuming an equilibrium between the reservoir and the nanochannel entrance, one can utilize the Boltzmann distribution to correlate the entrance values to the reservoir values. Assuming the left reservoir is grounded $\left(\varphi_{\text {leftres. }}=0\right)$ and the right reservoir is biased at voltage $v\left(\varphi_{\text {rightres. }}=v\right)$, we can write

$$
\begin{aligned}
& N_{1}=N_{\text {res }} \sqrt{1+\left(\frac{2 \sigma}{e h_{1} N_{\text {res }}}\right)^{2}}, \\
& N_{4}=N_{\text {res }} \sqrt{1+\left(\frac{2 \sigma}{e h_{2} N_{\text {res }}}\right)^{2}},
\end{aligned}
$$




$$
\begin{gathered}
\varphi_{1}=\frac{k_{b} T}{e} \frac{\sigma}{|\sigma|} \cos h^{-1}\left(\sqrt{1+\left(\frac{2 \sigma}{e h_{1} N_{r e s}}\right)^{2}}\right), \\
\varphi_{4}=v+\frac{k_{b} T}{e} \frac{\sigma}{|\sigma|} \cos h^{-1}\left(\sqrt{1+\left(\frac{2 \sigma}{e h_{2} N_{r e s}}\right)^{2}}\right) .
\end{gathered}
$$

Since $N_{\text {left }}, N_{\text {right }}, \varphi_{\text {right }}$, and $\varphi_{\text {left }}$ are known, we can directly find the values for $N_{1}, N_{4}, \varphi_{1}, \varphi_{4}$. The validity of this equilibrium assumption is contingent upon avoiding large currents and formation of the ionic concentration polarization (ICP) zones at the nanochannel entrances. At high voltages, ion depletion and enrichment zones are created in the anodic and cathodic sides of a negatively charged nanochannel, respectively. The regime of parameters below which the ICP is avoided can be found by calculating the limiting current, i.e., the diffusion limited current observed in the $i-v$ response of the nanochannel after it displays the early ohmic behaviour at smaller voltages. This matter has been the subject of a group of studies, and simple models for prediction of the limiting current have been presented. ${ }^{26,43}$ In this study, avoiding large voltages allows us to use Equation (5) with adequate accuracy (see the supplementary material for the complementary discussion).

There are six factors that dictate the diode characteristic of the stepped nanochannel, three of which are geometric, including channel height $h_{1}$, height ratio $\tau$, and the length ratio $l$, in addition to the surface charge, the electrolyte concentration, and the applied voltage. The aforementioned nondimensionalization allows us to see the above system of equation is only a function of the length ratio $l$ and not the channel length $(L)$. In fact, although the current is inversely proportional to the nanochannel length $(I=\bar{I} M=\bar{I} \bar{M} 2 \sigma D / e L)$, the rectification factor $\left(\left|\frac{I_{\text {forvardbias }}}{I_{\text {rearese has }}}\right|\right)$ remains the same for any length of channel as long as the length ratio $l$ does not change.

\section{B. Model validation}

Equations (1) and (2) and the associated boundary conditions were solved using the COMSOL Multiphysics Finite element Package v4.4. All parameters were varied, and the results obtained were compared against the 1D model (see the supplemental material, Section III for details of the FEM model). An excellent agreement between the full numerical solution and the 1D model was observed, with error typically being a few percent. The cross-section averaged concentration profiles and the electric potential along the nanochannel obtained from the 1D model closely mimics the FEM results (see the supplementary material, Section IV). Table I compares the forward bias current, reverse bias current, and the rectification factor obtained by these two models for a representative case of a stepped nanochannel with length $L=50 \mu \mathrm{m}$, $w=3 \mu \mathrm{m}, \sigma=-3 \mathrm{mC} / \mathrm{m}^{2}$, and $10 \mathrm{mM}$ salt concentration at $\pm 1 \mathrm{~V}$. This comparison is made to evaluate the $1 \mathrm{D}$ model and to ensure that it has been correctly implemented.

\section{NUMERICAL RESULTS}

The 1D model relies on the assumption of constant surface charge along the nanochannel. In addition, while the accumulation of ion at the step can cause a reduction in the diffusion coefficient, here the diffusion coefficient is assumed to be constant along the nanochannel. For all the calculations, the diffusion coefficient is calculated based on the Einstein relation, $D=\frac{\mu k_{b} T}{e}$, with $\mu=7.8 \times 10^{-8} \mathrm{~m}^{2} \mathrm{~V}^{-1} \mathrm{~s}^{-1}$.

The $i-v$ curve of a stepped nanochannel with $h_{1}=5 \mathrm{~nm}, \tau=20, L=600 \mu \mathrm{m}, l=0.1$, $\sigma=-3 \mathrm{mC} / \mathrm{m}^{2}$, and $0.01 \mathrm{M}$ electrolyte is shown in Fig. 2(a) which clearly demonstrates the current rectification. The rectification factor is also shown in Fig. 2(b) as a function of the applied voltage. The rectification increases with voltage and can reach values up to 38 at $5 \mathrm{~V}$. Further increasing the voltage is known to first cause a diffusion-limited current regime, and 
TABLE I. Comparison of the simulation results obtained by the 1D model and the FEM model for different heights, length ratios, and height ratios ( $L=50 \mu \mathrm{m}, w=3 \mu \mathrm{m}, \sigma=-3 \mathrm{mC} / \mathrm{m}^{2}$, and $10 \mathrm{mM}$ electrolyte).

\begin{tabular}{|c|c|c|c|c|c|c|c|c|}
\hline \multicolumn{3}{|c|}{ Geometric factor } & \multicolumn{2}{|c|}{ Forward bias current (nA) } & \multicolumn{2}{|c|}{ Reverse bias current (nA) } & \multicolumn{2}{|c|}{ Rectification factor } \\
\hline$h_{1}$ & $l$ & $\tau$ & 1D model & 2D FEM & 1D model & 2D FEM & 1D model & 2D FEM \\
\hline \multirow[t]{6}{*}{$5 \mathrm{~nm}$} & \multirow[t]{3}{*}{0.1} & 5 & -0.308 & -0.31 & 0.0614 & 0.0618 & 5.01 & 5.02 \\
\hline & & 10 & -0.664 & -0.661 & 0.071 & 0.0717 & 9.35 & 9.21 \\
\hline & & 15 & -0.879 & -0.869 & 0.081 & 0.082 & 10.85 & 10.6 \\
\hline & \multirow[t]{3}{*}{0.5} & 5 & -0.19 & -0.19 & 0.059 & 0.06 & 3.20 & 3.18 \\
\hline & & 10 & -0.195 & -0.194 & 0.069 & 0.07 & 2.80 & 2.77 \\
\hline & & 15 & -0.181 & -0.181 & 0.0795 & 0.0802 & 2.27 & 2.25 \\
\hline \multirow[t]{6}{*}{$10 \mathrm{~nm}$} & \multirow[t]{3}{*}{0.1} & 5 & -0.553 & -0.552 & 0.1317 & 0.133 & 4.20 & 4.13 \\
\hline & & 10 & -1.002 & -0.99 & 0.166 & 0.169 & 6.04 & 5.86 \\
\hline & & 15 & -1.254 & -1.234 & 0.201 & 0.204 & 6.24 & 6.04 \\
\hline & \multirow[t]{3}{*}{0.5} & 5 & -0.278 & -0.277 & 0.118 & 0.119 & 2.35 & 2.33 \\
\hline & & 10 & -0.278 & -0.278 & 0.146 & 0.148 & 1.90 & 1.88 \\
\hline & & 15 & -0.263 & -0.264 & 0.161 & 0.162 & 1.63 & 1.62 \\
\hline
\end{tabular}

then again there will be the overlimiting current due to creation of a strong flow. ${ }^{41,43}$ Such phenomena cannot be predicted by our model as the diffusion and the electrolyte flow from the reservoir to the nanochannel have not been accounted for. Addressing phenomena at such high voltage regimes is out of scope of this paper, and the parametric study of the rectification factor is performed at $1 \mathrm{~V}$ in the rest of paper. To explain the current rectification, the concentration of cations and anions as well as the electric potential along the nanochannel for the forward bias and reverse bias cases are plotted in Figs. 2(c) and 2(d). It is clear that the forward bias corresponds to significant accumulation of both cations and anions at the step of the nanochannel, while a concentration depletion zone occurs in the same location under the reverse bias. The accumulation and depletion result from different levels of selective ion transport in the shallow and deep side of the nanochannel. Because of a relatively thick electrical double layer (i.e., larger $\frac{\lambda_{d}}{h}, \lambda_{d}$ is the Debye screening length), the shallow side of the channel is more cation
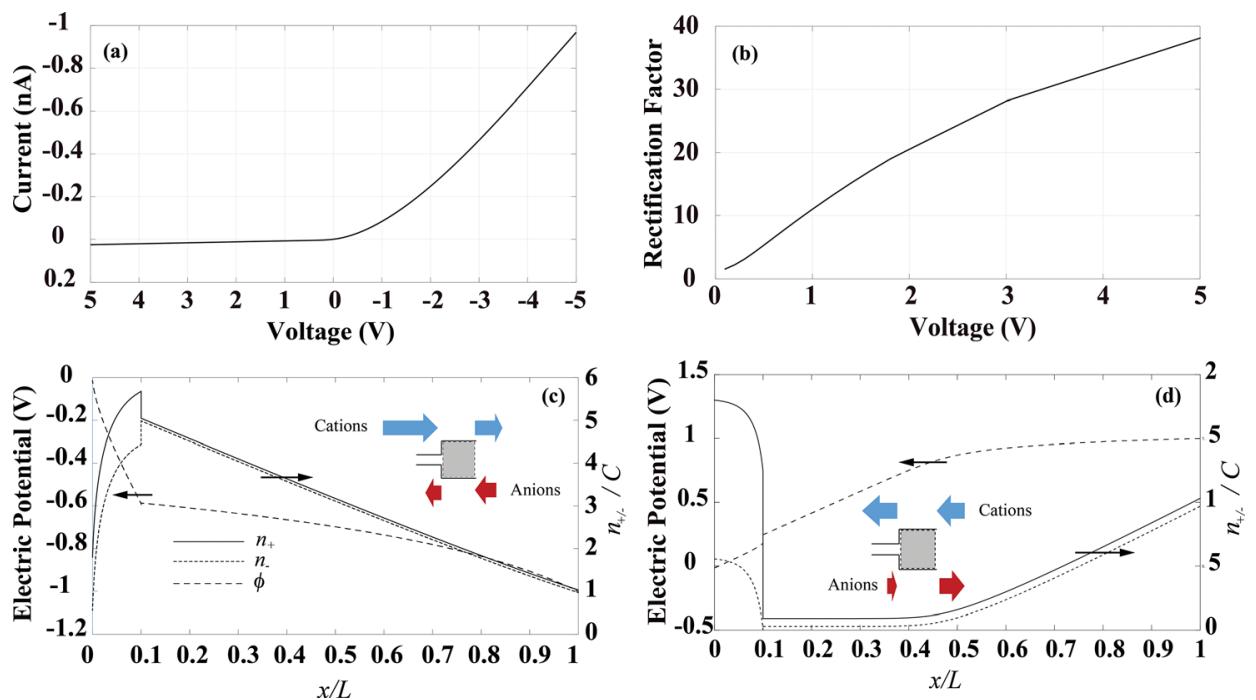

FIG. 2. (a) The $i-v$ curve and (b) the rectification factor as a function of applied bias for a stepped channel with $h_{1}=5 \mathrm{~nm}, \tau=20, L=600 \mu \mathrm{m}, l=0.1, \sigma=-3 \mathrm{mC} / \mathrm{m}^{2}$, and $0.01 \mathrm{M}$. (c) Cations, anions, and electric potential profiles along the nanochannel for the forward bias and (d) the reverse bias for $1 \mathrm{~V}$ applied voltage. 
selective than the deep side. This selectivity difference would temporarily result in an imbalance of ionic current of each ion type across the step when a bias is applied. Consequently, the cation current in the shallow side of the channel would be larger than that in the deep side and the anion current in the shallow side would be smaller than that in the larger side. Under a forward (or reverse) bias, this current imbalance will cause continuous accumulation (or depletion) of cation and anion at the step until the concentration gradient can compensate the existing imbalance of ionic current, which then leads to a steady-state.

Current rectification mechanism can be better understood by looking at the conductance as a function of electrolyte concentration. The nanochannel conductance $(G=i / v)$ and the rectification factor of a stepped nanochannel with $h_{1}=10 \mathrm{~nm}, \tau=10, L=600 \mu \mathrm{m}, l=0.2$, $\sigma=-3 \frac{m C}{m^{2}}$ as a function of electrolyte concentration are presented in Fig. 3(a). It is observed that rectification factor does not monotonically increase with the decreasing bulk concentration but shows a maximum value $\left(C_{\max }\right)$ at a concentration between $\frac{2|\sigma|}{e h_{2}}$ and $\frac{2|\sigma|}{e h_{1}}$. Such a behavior has been previously reported for single conical nanopores ${ }^{12}$ or membranes with such asymmetric pores. ${ }^{23}$ The existence of this maximum rectification factor can be explained again by the degree of selective ion transport in two sides of the nanochannel. At very low concentrations, electrical double layers from the top and bottom surfaces overlap in both the shallow side and the deep side of the nanochannel and the difference of cation selectivity across the step becomes small. Thus, this decreases the imbalance of ionic current for each ion type across the step and leads to reduced accumulation (depletion) when a forward (reverse) bias is applied and hence a small rectification. This is aligned with the known fact that nanochannel conductance at low concentration is not a function of channel height, and as such the height difference in the nanochannel is not sensed. On the other hand, at high concentrations where the thickness of the electrical double layer is much smaller than the height of both the shallow side and the deep side of the channel, both sides do not have much cation selectivity and current rectification diminishes as well. Therefore, the maximum rectification would occur at a concentration where the shallow channel is very cation-selective but the deep channel shows negligible selectivity. This concentration $\left(C_{\max }\right)$ is a function of surface charge and nanochannel geometry.
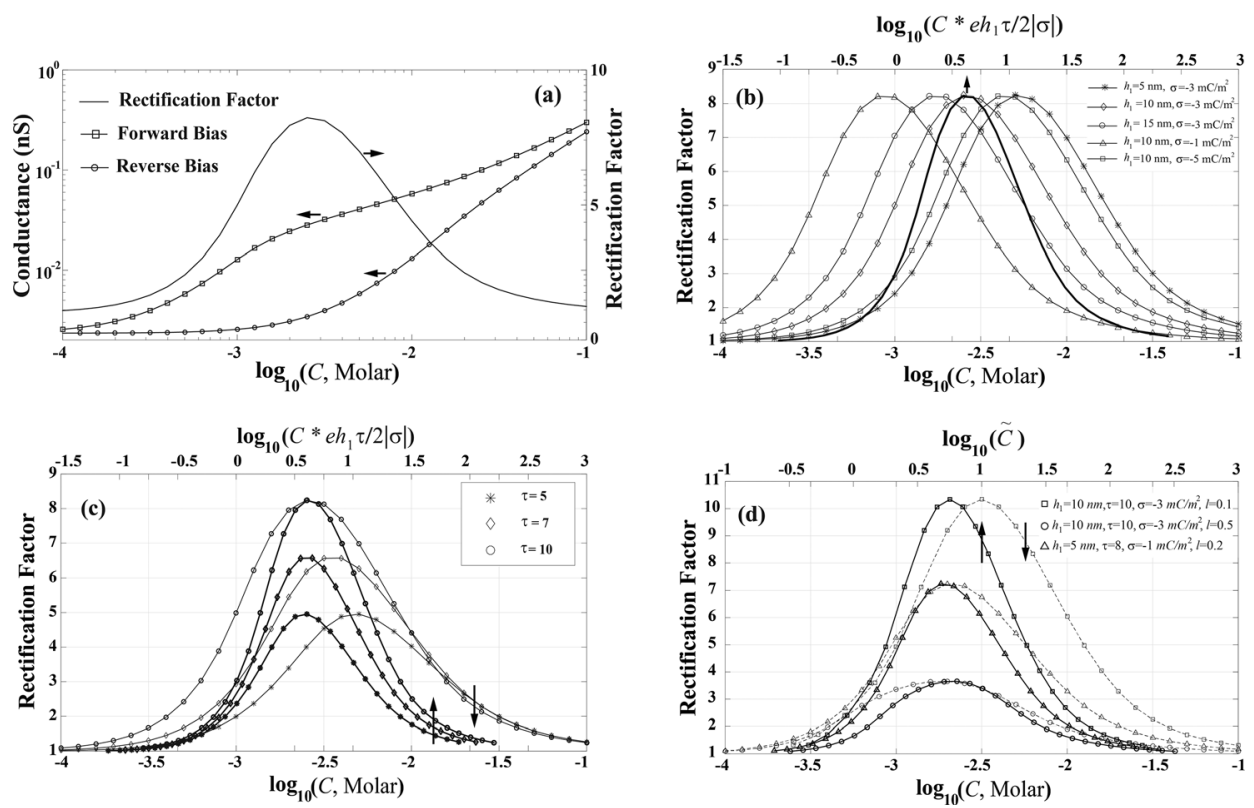

FIG. 3. (a) Forward bias and reverse bias conductance and rectification factor versus concentration for $h_{1}=10 \mathrm{~nm}, \tau=10$, $L=600 \mu \mathrm{m}, l=0.2$, and $\sigma=-3 \frac{\mathrm{mC}}{\mathrm{m}^{2}}$. (b) Rectification factor versus nondimensional concentration for different $h_{1}$ and $\sigma$ $(l=0.2$ and $\tau=10)$. (c) Effect of height ratio on $C_{\max }$ with all other factors kept constant $\left(h_{1}=10 \mathrm{~nm}, l=0.2\right.$, $\sigma=-3 \frac{\mathrm{mC}}{\mathrm{m}^{2}}$ ). (d) Effect of length ratio on $C_{\max }$ and comparison of the rectification factors of two nanofluidic diodes with different heights, height ratios, length ratios, and surface charges. 
Normalizing the concentration by $\left|\frac{2 \sigma}{e h_{1} \tau}\right|=\left|\frac{2 \sigma}{e h_{2}}\right|$ for different values of $\sigma$ and $h_{1}$ yields a universal curve for rectification factor shown in Fig. 3(b), if the height ratio $(\tau)$ and the length ratio (l) are kept constant. This is not unexpected and is consistent with our normalization in Equation (4). In the system of Equation (3) for a fixed applied bias, all the independent factors are summarized in three nondimensional parameters, namely, $\bar{N}, \tau$, and $l$. So if the height ratio and the length ratio do not change, then the nondimensional concentration $\bar{N}\left(=\frac{N}{2 \sigma / e h_{1}}\right)$ carries all the information, and hence the aforementioned normalization results in a universal curve. On the other hand, if the other two nondimensional geometric factors $(l$ and $\tau$ ) vary, they create a more complicated picture. Different height ratios dictate different levels of ion selectivity to the deep side of the nanochannel which in addition to changing the rectification factor, shifts the $C_{\max }$. This is demonstrated in Fig. 3(c) with thin lines where $h_{1}, l$, and $\sigma$ are kept constant and only the height ratio $(\tau)$ takes different values $\left(h_{1}=10 \mathrm{~nm}, l=0.2, \sigma=-3 \frac{\mathrm{mC}}{\mathrm{m}^{2}}\right)$. Nevertheless, as it can be seen the normalized $C_{\max }$ is not appreciably affected by variations of $\tau$ (thick lines), allowing us to predict $C_{\max }$ for arbitrary values of $h_{1}, h_{2}$, and surface charges. Finally, in Fig. 3(d), the effect of last nondimensional number and geometric factor, i.e., the length ratio $(l)$ is accounted for. The length ratio $(l)$ mainly manifests itself by altering the electric field along the nanochannel, and any change in the length ratio other than altering the peak rectification factor can shift the value of $C_{\max }$, too (compare the squares and circles with $l=0.1$ and $l=0.5$ plotted with thin lines in Fig. 3(d)). Nevertheless, by using $\frac{2|\sigma|}{e h_{1} \tau}(1-l)$ for concentration normalization, a very slight change in $C_{\max }$ is observed (thick lines). This normalization indicates that $C_{\max }$ is governed by the height and length of the deep side of the nanochannel. Through this normalization $\left(\tilde{C}=C * \frac{e h_{1} \tau}{2 \mid \sigma(1-l)}\right)$, we can combine all three nondimensional numbers to provide an estimation of the electrolyte concentration associated with maximum rectification for any of such nanofluidic diodes. This can be better observed by comparing the squares and triangles in Fig. 3(d) where two completely different geometries and surface charges yield the same $\tilde{C}_{\max }$. Use of this proposed nondimensional concentration, $\tilde{C}$, facilitates the design and prediction of rectification of this type of nanofluidic diodes.

Further numerical analysis yields the optimal length ratio and height ratio for maximum rectification. Fig. 4(a) presents the rectification factor as a function of the nondimensional concentration and the length ratio with a height ratio of $\tau=10$. It can be observed that rectification is maximized when length of the shallow side is less than one tenth of the entire nanochannel. Larger length ratios would decrease the rectification, and very small length ratios also would sharply reduce the rectification factor. This observation can be explained on the basis of the resistance of the shallow and deep sides of the nanochannel. At any given height ratio, a large length ratio causes the resistance of the shallow nanochannel to dominate the transport and a diode starts to act more like a resistor (rectification factor close to 1). A similar scenario can also occur for very small length ratios, where the resistance of the shallow side becomes insignificant compared with the deep side. Same logic can be employed to explain how (for a constant height ratio) at large length ratios the peak rectification shifts to smaller nondimensional concentrations $\left(\bar{N}_{\max }\right)$, and the $l_{\max }$ (length ratio at which maximum rectification occurs) for smaller nondimensional concentration shifts to larger values. Fig. 4(b) shows the interplay between the length ratio and the height ratio for a constant nondimensional concentration $(\bar{N}=4.8)$. For each length ratio, the peak rectification occurs at a certain height ratio $\left(\tau_{\max }\right)$ and this $\tau_{\max }$ for smaller length ratios shifts to larger values. In fact, a large height ratio causes the resistance of the shallow nanochannel to dominate and decreasing the length ratio brings back the balance between the resistances of the two sides. It can be seen that smaller length ratios along with larger height ratios yield larger rectifications. Finally, the study of rectification factor versus the nondimensional concentration and the height ratio for a constant length ratio $(l=0.2)$ is presented in Fig. 4(c). Very small height ratios depreciate the ion selectivity contrast between the shallow and deep sides of the nanochannel which results in small rectification factors. Very large height ratios on the other hand make the resistance of the deep side negligible compared with the shallow side, and a stepped nanochannel acts similar to its shallow side. Therefore, for any nondimensional concentration, there is an optimal height ratio for which the rectification factor is maximum. This $\tau_{\max }$ for smaller values of concentration shifts to larger 

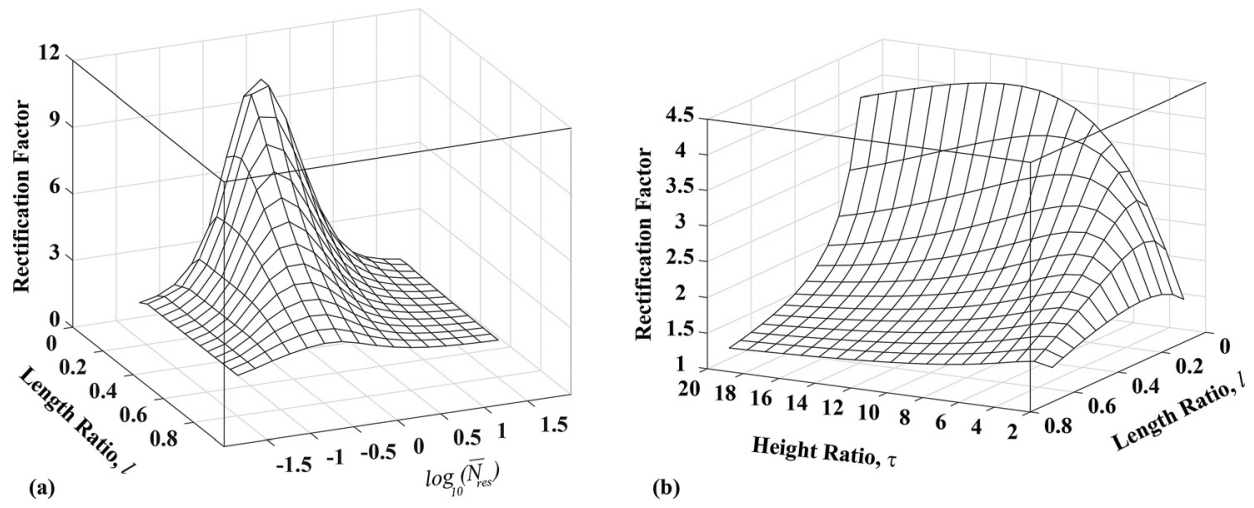

(b)

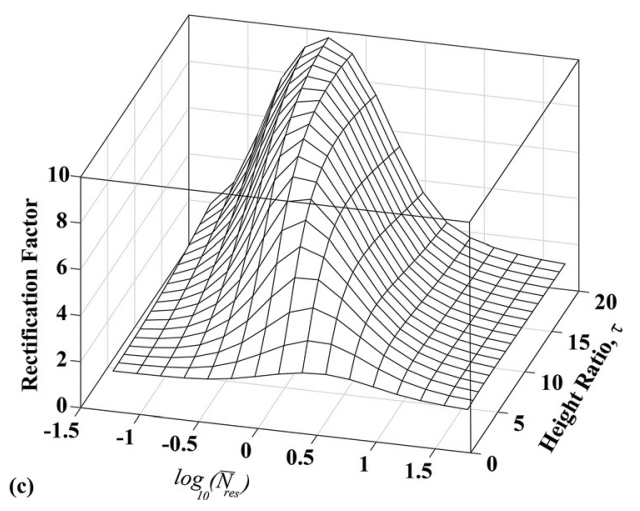

FIG. 4. (a) Rectification factor against the length ratio and nondimensional concentration for a constant height ratio of $\tau=10$. (b) Rectification factor against length ratio and height ratio for a constant nondimensional concentration of $\bar{N}=4.8$. (c) Rectification factor against height ratio and nondimensional concentration for a constant length ratio of $l=0.2$. All calculations performed at $\pm 1 \mathrm{~V}$ applied bias.

values to ensure the deep side is not as perm selective as the shallow side. Figs. 4(a) and 4(c) demonstrate that by increasing the length and height ratios, the $\bar{N}_{\max }$ (nondimensional concentration at which maximum rectification occurs) shifts to smaller values, and introduction of the $\frac{\tau}{1-l}$ term in $\tilde{C}$ in Figs. 3(c) and 3(d) was to account for these shifts.

\section{EXPERIMENTAL RESULTS}

\section{A. Materials and methods}

Stepped nanochannels were fabricated using the standard etching and bonding method. ${ }^{44}$ The stepped nanochannels were fabricated by two steps photolithography, each followed by the reactive ion etching. Before the second photoresist is spun-coat, the first layer must be hard baked over night at $120^{\circ} \mathrm{C}$ to ensure it remains unaffected by the second UV exposure and maintains its structural stability during second development. Width of both sides of the nanochannels is $3 \mu \mathrm{m}$, the shallow side is $L_{1}=50 \mu \mathrm{m}$ long, and the deep side is $L_{2}=550 \mu \mathrm{m}$ long. After etching the nanochannels, two microchannels each $6 \mathrm{~mm}$ long, $1 \mathrm{~mm}$ wide and $40 \mu \mathrm{m}$ deep were etched using DRIE on both terminals of the nanochannels and four $2 \mathrm{~mm} \times 2 \mathrm{~mm}$ through holes later etched using DRIE serving as the reservoirs. Finally, $300 \mathrm{~nm}$ thick dry thermal oxide layer was grown on the silicon chips, and the chips were cleaned with Piranha $(3: 1$, $\mathrm{H}_{2} \mathrm{SO}_{4}: \mathrm{H}_{2} \mathrm{O}_{2}$ ) and bonded to a Borofloat glass by using anodic bonding at $400^{\circ} \mathrm{C}$ and $350 \mathrm{~V}$. A microscope image of a chip used for the experiments along with an AFM image of the location of the steps are shown in Figs. 5(a) and 5(b). Each chip contains 30 stepped nanofluidic channel with $h_{1}=16 \mathrm{~nm}$ and $h_{2}=110 \mathrm{~nm}$. A Keithley 6430 source meter (Keithley Instruments, Inc.) controlled by a Matlab program was used for the conductance measurements. All the 

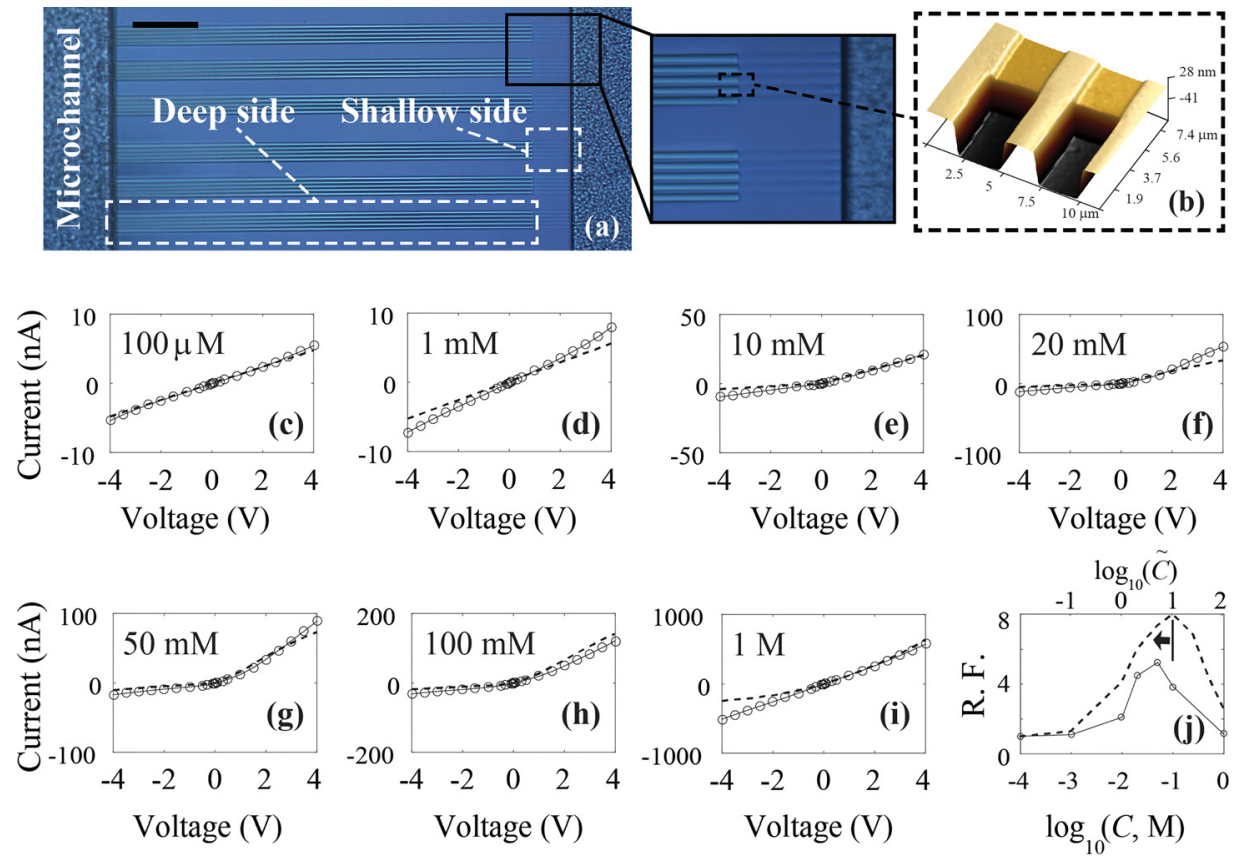

Simulations

Experimental Results

FIG. 5. (a) Microscope and (b) AFM images of a stepped channel used for experiment. The scale bar in (a) is $100 \mu \mathrm{m}$. (c)-(i) The measured $i-v$ curves (solid lines) for $100 \mu \mathrm{M}, 1 \mathrm{mM}, 10 \mathrm{mM}, 20 \mathrm{mM}, 50 \mathrm{mM}, 100 \mathrm{mM}$, and $1 \mathrm{M} \mathrm{KCl}$ concentrations compared against theoretical results. (Dashed lines) (j) Experimental and theoretical rectification factor versus concentration at $4 \mathrm{~V}$.

measurements were performed with the Potassium Chloride solutions using Silver/Silver Chloride electrodes in a dark Faraday cage to isolate the measurements from any external electromagnetic waves and electric fields. The $\mathrm{pH}$ value of the solutions was measured to be 6 . Measurements were carried using a linear sweep sequence from 0 to $4 \mathrm{~V}$ with $20 \mathrm{~s}$ source delay to ensure a steady state readout. Before the measurement, $20 \mathrm{~min}$ oxygen plasma was applied to the chips to create super-hydrophilic surfaces and thus to avoid any gas entrapment in the nanochannels.

\section{B. Measurements}

The voltage-current curves for seven concentrations ranging from $100 \mu \mathrm{M}$ to $1 \mathrm{M}$ are shown in Figs. 5(c)-5(i). In each plot, the solid line is the experimental results and the dashed line is the theoretical prediction. In order to compare the experimental results with theory, in particular, at higher concentrations, it is important to account for decrease in the ionic mobility at higher concentrations. For full dissociated ions such as $\mathrm{K}^{+}$and $\mathrm{Cl}^{-}$, ionic mobility in a bulk solution can be expressed as ${ }^{45}$

$$
\mu=\frac{1}{1+\frac{c_{1} z \sqrt{I_{z}}}{1+c_{2} a \sqrt{I_{z}}}} \mu_{\infty},
$$

where $I_{z}=\frac{1}{2} \sum_{j} z_{j}^{2} n_{j}$ is the ionic strength, and $c_{1}=1.825(\varepsilon T)^{-3 / 2}$ and $c_{2}=50.3(\varepsilon T)^{-3 / 2}$ depend on the absolute temperature and the dielectric constant of the solvent. For water at $25^{\circ} \mathrm{C}, c_{1}=0.508 \mathrm{M}^{-1 / 2}$ and $c_{2}=3.29 \mathrm{~nm}^{-1} \mathrm{M}^{-1 / 2}$. Finally, $a$ is an adjustable parameter related to the ion size which for $\mathrm{K}^{+}$and $\mathrm{Cl}^{-}$is equal to a $\approx 0.3 \mathrm{~nm}$. Mobilities of $\mathrm{K}^{+}$and $\mathrm{Cl}^{-}$ in infinite-dilution are $\mu_{\infty, K^{+}}=7.6 \times 10^{-8} \mathrm{~m}^{2} \mathrm{~V}^{-1} \mathrm{~s}^{-1}$ and $\mu_{\infty, C l^{-}}=7.91 \times 10^{-8} \mathrm{~m}^{2} \mathrm{~V}^{-1} \mathrm{~s}^{-1}$, respectively. According to this equation, the ionic mobility of $\mathrm{KCl}$ at $1 \mathrm{M}$ is about $66 \%$ of that at infinite-dilution. 
The experimental results are in agreement with the theoretical results, although there are some discrepancies observed, too. The rectification factor as a function of concentration illustrates a trend expected from the model (Fig. 5(j)) with a peak rectification at $50 \mathrm{mM}$. Nevertheless, the theoretical peak rectification has shifted to higher values of normalized concentration compared with the experiments, suggesting that surface charge has been overestimated. To obtain the surface charge, the theoretical $i-v$ curve was fit to the $100 \mu \mathrm{M}$ results and the value of $\sigma=-50 \mathrm{mC} / \mathrm{m}^{2}$ was obtained. While this fairly high measured surface charge is consistent with previous measurements in a similar nanochannel ${ }^{40}$ this value can only serve as an upper limit for the surface charge. In fact, when fitting a curve to the $100 \mu \mathrm{M}$ results, we ignored the electroosmosis effect which can be as important as the electrophoretic term at low concentrations. ${ }^{6,42}$ This results in overestimation of the surface charge which in turn would shift the rectification factor curve in Fig. $5(\mathrm{j})$ to the right. In fact, this design of nanofluidic diode enables us to estimate the value of surface charge without relying on $i-v$ curves at low concentrations. Based on the peak rectification location $\left(C_{\max }\right)$, the surface charge cannot be higher than $\sim-20 \mathrm{mC} / \mathrm{m}^{2}$. Our estimation of the electroosmotic flow based on uniform height channels also indicates that electroosmosis can contribute to $\sim 30 \%$ of the total current at low concentrations, which means the surface charge at $100 \mu \mathrm{M}$ has been overestimated by at least $30 \%$ (see the supplementary material, Section IV). At high electrolyte concentrations $(50 \mathrm{mM}, 100 \mathrm{mM}$, and $1 \mathrm{M}$ ) where the electroosmosis becomes negligible compared with the electrophoretic term, the surface charge of $-20 \mathrm{mC} / \mathrm{m}^{2}$ in the $1 \mathrm{D}$ model better predicts the experimental results; however, it causes significant deviation from experiments at lower concentrations (results not shown). It is noticed that the experimental rectification factor is smaller than the theoretical values. We believe this is also partly due to the electroosmosis effect which reduces the rectification mainly by increasing the absolute value of reverse bias current, although it increases the forward bias current too. Another shortcoming of the present analysis is the assumption of constant surface charge throughout the entire nanochannel and for different concentrations. It is known that this assumption does not reflect the complete physics and a more sophisticated model accounting for dissociation of $\mathrm{SiOH}$ groups better predicts the experimental results. ${ }^{46-48}$ However, implementation of this model in our simulation is not easily possible and makes the system of equation very complicated, and hence is avoided. Nonetheless, the constant surface charge assumption allowed us to reliably predict the experimental results.

\section{CONCLUSIONS}

In summary, we presented a new method for ionic current rectification in nanofluidic systems. The advantages of this method over previously reported methods are (i) it does not require any surface modification and thus is very stable and easy to work with and (ii) it can very easily be integrated with other fluidic elements in 2D lab-on-a-chip fluidic circuits. Additionally, the design of these nanofluidic diodes is facilitated by knowledge of the nondimensional concentration that dictates its rectification behavior. It was observed that the rectification factor does not monotonically increase by decreasing the electrolyte concentration, rather it reaches a peak in midrange concentration. The location of this peak is determined by the surface charge density and the nanochannel dimensions, and hence this type of nanofluidic diode can be configured to yield high rectifications at high electrolyte concentrations. Future studies will focus on electroosmotic flow in stepped nanochannels and its effect on the electrokinetics in such systems.

\section{SUPPLEMENTARY MATERIAL}

See supplementary material for the derivation of a 1-D model based on PNP equation, discussion of ionic concentration polarization (ICP), the finite element model (FEM), comparison of the 1-D Model and the FEM profiles, and contribution of electroosmosis to the current. 


\section{ACKNOWLEDGMENTS}

This work was supported by the Faculty Startup Fund (Boston University, USA) and the American Chemical Society Petroleum Research Fund (ACS PRF\# 54118-DNI7). The authors would like to thank the photonics Center at Boston University for the use of their fabrication and characterization facilities. B.L. is grateful to the international education program for Tsien Excellence in Education Program (TEEP) at Tsinghua University for supporting his visit to Boston University.

${ }^{1}$ A. Alcaraz, P. Ramírez, E. García-Giménez, M. L. Lopez, A. Andrio, and V. M. Aguilella, J. Phys. Chem. B 110(42), 21205-21209 (2006)

${ }^{2}$ H. Miedema, M. Vrouenraets, J. Wierenga, W. Meijberg, G. Robillard, and B. Eisenberg, Nano Lett. 7(9), 2886-2891 (2007).

${ }^{3}$ E. García-Giménez, A. Alcaraz, V. M. Aguilella, and P. Ramírez, J. Membr. Sci. 331(1), 137-142 (2009).

${ }^{4}$ Y. Zhang, J. Clausmeyer, B. Babakinejad, A. López Córdoba, T. Ali, A. Shevchuk, Y. Takahashi, P. Novak, C. Edwards, and M. Lab, ACS Nano 10(3), 3214 (2016).

${ }^{5}$ E. B. Kalman, I. Vlassiouk, and Z. S. Siwy, Adv. Mater. 20(2), 293-297 (2008).

${ }^{6} \mathrm{H}$. Daiguji, P. Yang, and A. Majumdar, Nano Lett. 4(1), 137-142 (2004).

${ }^{7}$ H. Daiguji, Y. Oka, and K. Shirono, Nano Lett. 5(11), 2274-2280 (2005).

${ }^{8}$ R. Karnik, K. Castelino, and A. Majumdar, Appl. Phys. Lett. 88(12), 123114 (2006).

${ }^{9}$ R. Karnik, R. Fan, M. Yue, D. Li, P. Yang, and A. Majumdar, Nano Lett. 5(5), 943-948 (2005).

${ }^{10}$ Z. Slouka, S. Senapati, and H.-C. Chang, Annu. Rev. Anal. Chem. 7, 317-335 (2014).

${ }^{11}$ Y. Liu and L. Yobas, Biosens. Bioelectron. 50, 78-83 (2013).

${ }^{12}$ I. Vlassiouk, T. R. Kozel, and Z. S. Siwy, J. Am. Chem. Soc. 131(23), 8211-8220 (2009).

${ }^{13}$ Z. Siwy, P. Apel, D. Baur, D. D. Dobrev, Y. E. Korchev, R. Neumann, R. Spohr, C. Trautmann, and K.-O. Voss, Surf. Sci. 532-535, 1061-1066 (2003).

${ }^{14}$ Z. S. Siwy, Adv. Funct. Mater. 16(6), 735-746 (2006).

${ }^{15}$ D. Constantin and Z. S. Siwy, Phys. Rev. E 76(4), 041202 (2007).

${ }^{16}$ J. Cervera and B. Schiedt, EPL (Europhys. Lett.) 71(1), 35 (2005).

${ }^{17}$ Z. Siwy, Y. Gu, H. Spohr, D. Baur, A. Wolf-Reber, R. Spohr, P. Apel, and Y. Korchev, EPL (Europhys. Lett.) 60(3), 349 (2002).

${ }^{18}$ Z. Siwy, P. Apel, D. Dobrev, R. Neumann, R. Spohr, C. Trautmann, and K. Voss, Nucl. Instrum. Methods Phys. Res. Sect. B 208, 143-148 (2003).

${ }^{19}$ C. Wei, A. J. Bard, and S. W. Feldberg, Anal. Chem. 69(22), 4627-4633 (1997).

${ }^{20}$ I. Vlassiouk and Z. S. Siwy, Nano Lett. 7(3), 552-556 (2007).

${ }^{21}$ Z. Siwy, E. Heins, C. C. Harrell, P. Kohli, and C. R. Martin, J. Am. Chem. Soc. 126(35), 10850-10851 (2004).

${ }^{22}$ P. Y. Apel, Y. E. Korchev, Z. Siwy, R. Spohr, and M. Yoshida, Nucl. Instrum. Methods Phys. Res. Sect. B 184(3), 337-346 (2001).

${ }^{23}$ P. Y. Apel, I. Blonskaya, N. Levkovich, and O. Orelovich, Pet. Chem. 51(7), 555-567 (2011).

${ }^{24}$ I. Vlassiouk, S. Smirnov, and Z. Siwy, ACS Nano 2(8), 1589-1602 (2008).

${ }^{25}$ R. Yan, W. Liang, R. Fan, and P. Yang, Nano Lett. 9(11), 3820-3825 (2009).

${ }^{26}$ R. Karnik, C. Duan, K. Castelino, H. Daiguji, and A. Majumdar, Nano Lett. 7(3), 547-551 (2007).

${ }^{27}$ Y. Green, Y. Edri, and G. Yossifon, Phys. Rev. E 92(3), 033018 (2015).

${ }^{28}$ L.-J. Cheng and L. J. Guo, ACS Nano 3(3), 575-584 (2009).

${ }^{29}$ K. P. Singh, K. Kumari, and M. Kumar, Appl. Phys. Lett. 99(11), 113103 (2011)

${ }^{30}$ L. Li, J. Mo, and Z. Li, Phys. Rev. Lett. 115(13), 134503 (2015).

${ }^{31}$ W. Guan, R. Fan, and M. A. Reed, Nat. Commun. 2, 506 (2011).

${ }^{32}$ L.-J. Cheng and L. J. Guo, Nano Lett. 7(10), 3165-3171 (2007).

${ }^{33}$ J.-Y. Jung, P. Joshi, L. Petrossian, T. J. Thornton, and J. D. Posner, Anal. Chem. 81(8), 3128-3133 (2009).

${ }^{34}$ G. Yossifon, Y.-C. Chang, and H.-C. Chang, Phys. Rev. Lett. 103(15), 154502 (2009).

${ }^{35}$ S. Mafe and P. Ramirez, Acta Polym. 48(7), 234-250 (1997).

${ }^{36}$ A. A. Sonin and G. Grossman, J. Phys. Chem. 76(26), 3996-4006 (1972).

${ }^{37}$ G. Sun, S. Senapati, and H.-C. Chang, Lab Chip 16(7), 1171-1177 (2016).

${ }^{38}$ Y. Yan, L. Wang, J. Xue, and H.-C. Chang, J. Chem. Phys. 138(4), 044706 (2013).

${ }^{39}$ Y. Yan, J. Schiffbauer, G. Yossifon, and H.-C. Chang, J. Chem. Phys. 143(22), 224705 (2015).

${ }^{40}$ D. Stein, M. Kruithof, and C. Dekker, Phys. Rev. Lett. 93(3), 035901 (2004).

${ }^{41}$ S. J. Kim, Y.-C. Wang, J. H. Lee, H. Jang, and J. Han, Phys. Rev. Lett. 99(4), 044501 (2007).

${ }^{42}$ D. G. Haywood, Z. D. Harms, and S. C. Jacobson, Anal. Chem. 86(22), 11174-11180 (2014).

${ }^{43}$ G. Yossifon, P. Mushenheim, Y.-C. Chang, and H.-C. Chang, Phys. Rev. E 79(4), 046305 (2009).

${ }^{44}$ M. A. Alibakhshi, Q. Xie, Y. Li, and C. Duan, Sci. Rep. 6, 24936 (2016).

${ }^{45}$ F. Baldessari, J. Colloid Interface Sci. 325(2), 526-538 (2008).

${ }^{46}$ S. H. Behrens and D. G. Grier, J. Chem. Phys. 115(14), 6716-6721 (2001).

${ }^{47}$ M. B. Andersen, H. Bruus, J. P. Bardhan, and S. Pennathur, J. Colloid Interface Sci. 360(1), 262-271 (2011).

${ }^{48}$ R. M. Smeets, U. F. Keyser, D. Krapf, M.-Y. Wu, N. H. Dekker, and C. Dekker, Nano Lett. 6(1), 89-95 (2006). 